LBNL-42972

SCMAG-659

\title{
A Four Cell Lattice for the UCLA Compact Light Source Synchrotron
}

\author{
A. A. Garren*, and M. A. Green** \\ * UCLA Center for Advanced Accelerators, Los Angeles CA 90095 \\ ** Lawrence Berkeley National Laboratory, Berkeley CA 94720, USA
}

March 1999

\section{Particle Accelerator Conference} New York NY, 29 March to 2 April 1999

To be Published in the PAC-99 Proceedings

* This work was performed with the support of the Regents of the University of California 


\title{
A Four Cell Lattice for the UCLA Compact Light Source Synchrotron
}

\author{
A. A. Garren*, and M. A. Green** \\ * UCLA Center for Advanced Accelerators, Los Angeles CA 90095 \\ ** Lawrence Berkeley National Laboratory, Berkeley CA 94720, USA
}

\section{Abstract}

The $1.5 \mathrm{GeV}$ compact light source UCS proposed for UCLA must fit into a shielded vault that is 9.144 meters (30 feet) wide. In order for the machine fit into the into the allowable space, the ring circumference must be reduced 36 meters, the circumference of the six cell lattice, to something like 26 or 27 meters. The four cell lattice described in this report has a ring circumference of 27.0 meters. The four cell ring consists of twelve $6.5 \mathrm{~T}$ superconducting dipoles, twenty-four conventional quadrupoles, sixteen conventional sextupoles and four straight sections 1.02 meters long. The $1.5 \mathrm{GeV}$ electron beam is bend in the dipoles to produce $\mathrm{x}$ rays with a critical energy of $9.8 \mathrm{keV}$. The superconducting dipoles have been modeled in three dimensions. The 3-D modeling shows that magnetic induction falls off from its full design value to nearly zero in about $50 \mathrm{~mm}$ at the ends of the dipoles. The magnetic length of the dipole is Calulated to be $403 \mathrm{~mm}$.

\section{BACKGROUND}

The six cell UCLA compact storage ring described in the 1997 Particle Accelerator Conference paper[1] appeared to be very good for producing intense high energy $\mathrm{X}$ rays. The six cell lattice had a low emittance and it tracked very well. The problem with six cell storage ring was the fact that it does not fit in an existing vault in the basement of a new building on the UCLA campus. The vault in the basement of building can not be enlarged to accommodate the six cell storage ring. As a result, the ring circumference must be reduced so that it can fit within a vault that is 9.142 meters ( 30 feet) wide. The length of the shielded vault is nearly 40 meters. This appears to be adequate for housing a ring injector and a number of experimental $\mathrm{X}$ ray beams. If the ring diameter can be reduced so the machine will fit in the vault, the new building at UCLA is adequate for the UCS ring.

Reducing the number of cells in the storage ring was the approach that was chosen to reduce the ring size. Three cell and four cell lattices were studied. Lattices with fewer numbers of cells have less space occupied by conventional quadrupoles and sextupoles. Ring circumference was also reduced by reducing the number of long straight sections. The object of the study was to reduce the number of the cells in the machine while trying to keep the beam emittance low.

\footnotetext{
* This work was supported by the Regents of the University of California.

+ email: magreen@lbl.gov
}

\section{THE REDUCED SIZED RING}

The designs for the reduced diameter storage rings were driven by the desire to extract synchrotron $\mathrm{X}$ rays from all of the dipoles. Like the six cell machine described in Ref [1], there are twelve superconducting bending magnets where the high energy $X$ rays are generated. Like the six cell machine, there was also a strong desire to reduce machine emittance so that the vacuum chamber throughout the ring can have the same cross-section. As a result, the quadrupoles and sextupoles would all have the same aperture. The number of types of quadrupoles could be reduced to two and there would be only one type of sextupole. The reduced emittance would lead to a smaller beam size in the dipoles and brighter $\mathrm{x}$ ray beams.

The six cell ring had six identical 6 meter long cells. Each of the six cells contains two 30 degree superconducting dipoles. Each cell has a 1.34 meter long drift space (between two quadrupole doublets) and a section that contains all of the chromaticity sextupoles. The sextupoles surround QF3 focusing quadrupole, which forces the dispersion to be zero in the long straight section. The dipoles have parallel faces that provide vertical edge-focusing.

The four cell ring studied had four identical cells that were 6.75 meters long. Each cell contains three 30 degree superconducting dipoles, a 1.02 meter long drift space (between two quadrupole doublets) and two sections that contain all of the chromaticity sextupoles. A pair of sextupoles surround the two QF3 focusing quadrupoles, which forces the dispersion to be zero in the long straight section. Defocusing is provided by the dipole parallel faces that provide vertical edge-focusing. The four cell ring computer simulation converged immediatêty.

A three cell ring with 8 meter long cells was studied. Each cell contained one 1.02 meter long straight section, two 0.5 meter long straight sections, four 30 degree superconducting dipoles, seven conventional quadrupoles and four conventional sextupoles. This ring would fit in the UCLA vault in all orientations. Unfortunately, the ring design studied did not work when modeled on the computer.

Figure 1 compares the half of the six cell ring (which is 12.5 meters wide) with half of the four cell ring (which is 9.1 meters wide). The six cell ring clearly does not fit in a hall that is 30 feet wide, whereas the four cell ring fits, but the margin is slim. Table 1 presents the magnet parameters for the six cell and four cell rings. Table 2 compares the calculated lattice parameters for six cell and four cell compact storage rings. 


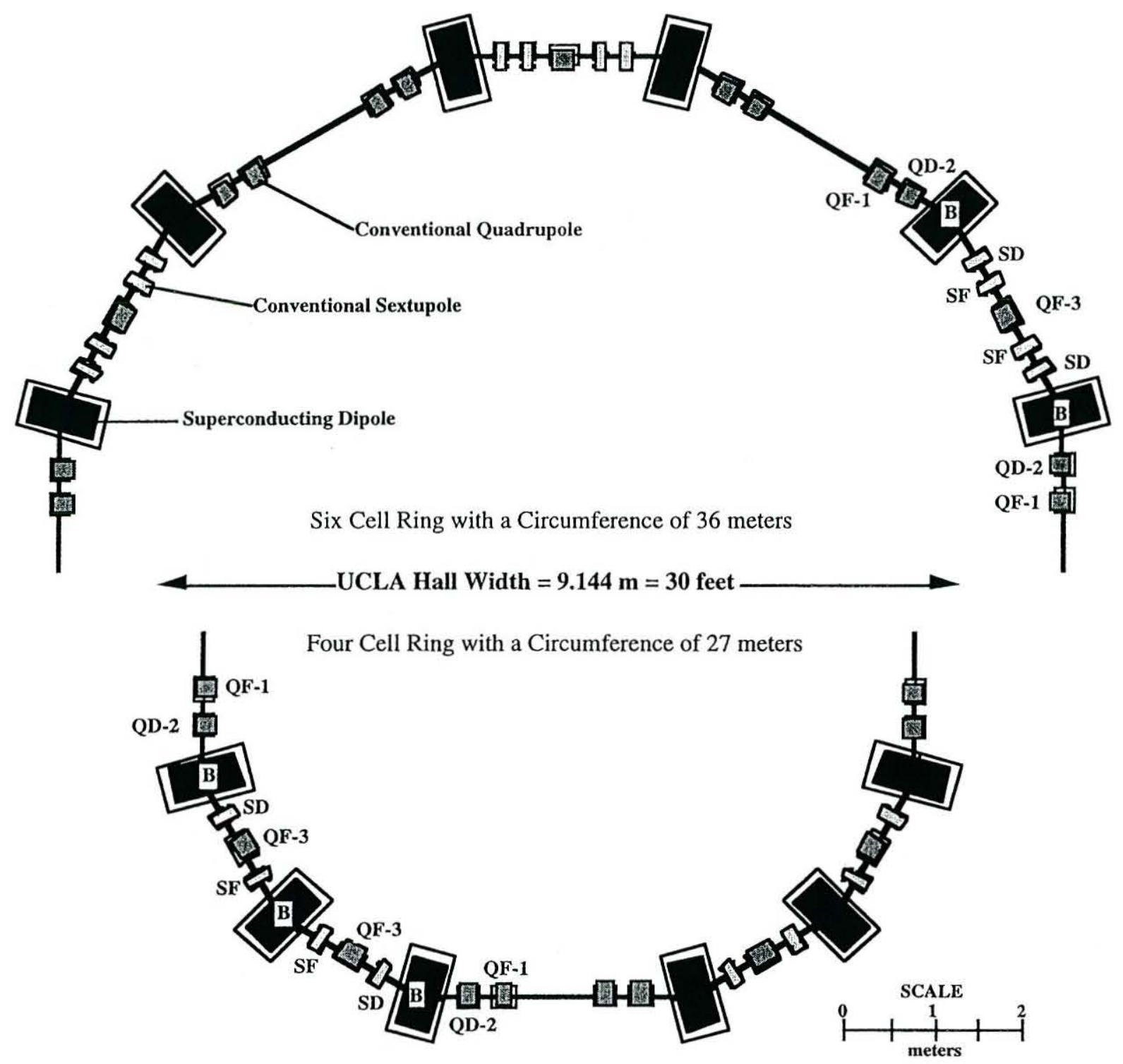

Figure 1: A comparison of Half of a Six $1.5 \mathrm{GeV}$ Compact Storage Ring with Half of a Four Cell Ring

Table 1: Magnet Parameters for the Six Cell and Four Cell Rings

\begin{tabular}{|c|c|c|c|c|c|c|}
\hline \multirow[b]{2}{*}{$\begin{array}{l}\text { Magnet } \\
\text { Type }\end{array}$} & \multicolumn{3}{|c|}{ Six Cell Storage Ring } & \multicolumn{3}{|c|}{ Four Cell Storage Ring } \\
\hline & $\begin{array}{c}\text { Strength } \\
\left(\mathrm{T}, \mathrm{Tm}^{-1}, \mathrm{Tm}^{-2}\right)\end{array}$ & $\begin{array}{l}\text { Aperture Dia. } \\
(\mathrm{mm})\end{array}$ & $\begin{array}{l}\text { M Length } \\
(\mathrm{mm})\end{array}$ & $\begin{array}{c}\text { Strength } \\
\left(\mathrm{T}, \mathrm{Tm}^{-1}, \mathrm{Tm}^{-2}\right)\end{array}$ & $\begin{array}{l}\text { Aperture Dia } \\
(\mathrm{mm})\end{array}$ & $\begin{array}{c}\text { M Length } \\
(\mathrm{mm})\end{array}$ \\
\hline \multicolumn{7}{|c|}{ uperconducting Dipole $(\mathrm{T})$} \\
\hline B & 6.468 & $40-180$ & 405 & 6.468 & $40-180$ & 405 \\
\hline \multicolumn{7}{|c|}{ Conventional Quadrupole $\left(\mathrm{T} \mathrm{m}^{-1}\right)$} \\
\hline QF-1 & 26.54 & 33.2 & 200 & 35.17 & 33.2 & 200 \\
\hline QD-2 & -19.54 & 33.2 & 200 & -36.00 & 33.2 & 200 \\
\hline QF-3 & 29.47 & 33.2 & 300 & 36.19 & 33.2 & 250 \\
\hline \multicolumn{7}{|c|}{ onventional Sextupole $\left(\mathrm{T} \mathrm{m}^{-2}\right)$} \\
\hline SF & 1279.3 & 33.2 & 100 & 1788.0 & 33.2 & 120 \\
\hline SD & -1695.0 & 33.2 & 100 & -1419.4 & 33.2 & 120 \\
\hline
\end{tabular}


Table 2: Lattice Parameters for the Six Cell and a Four Cell 1.5 GeV Compact Rings

\begin{tabular}{|c|c|c|}
\hline PARAMETER & $\begin{array}{c}6 \text { Cell } \\
\text { Ring }\end{array}$ & $\begin{array}{c}4 \text { Cell } \\
\text { Ring }\end{array}$ \\
\hline Maximum Design Energy (GeV) & 1.5 & 1.5 \\
\hline Injection Energy $(\mathrm{GeV})$ & 0.3 & 0.3 \\
\hline Max. Design Beam Current (mA) & 200 & 200 \\
\hline Storage Ring Circumference (m) & 36.0 & 27.0 \\
\hline Dipole Bend Radius (mm) & 773.5 & 773.5 \\
\hline X-ray Critical Energy* (keV) & 9.78 & 9.78 \\
\hline Number of X-ray Sources & 12 & 12 \\
\hline Extracted X-ray Power* (kW) & 123.2 & 123.2 \\
\hline $\mathrm{X}$-ray Brightness $* *\left(\mathrm{MWm}^{-2}\right)$ & 3.42 & 3.94 \\
\hline Stored Electron Beam Energy* (J) & 36.1 & 27.1 \\
\hline No. of Long Straight Sections & 6 & 4 \\
\hline Long Straight Length (m) & 1.34 & 1.02 \\
\hline Number of Cells & 6 & 4 \\
\hline No. of S/C Dipoles per Cell & 2 & 3 \\
\hline No. of Quadrupoles per Cell & 5 & 6 \\
\hline No. of Sextupoles per Cell & 4 & 4 \\
\hline Horz. Op. Emittance* (nm) & 309 & 475 \\
\hline Vert. Op. Emittance* (nm) & 34 & 53 \\
\hline Horizontal Tune & 4.42 & 4.18 \\
\hline Vertical Tune & 2.38 & 2.69 \\
\hline Horizontal Chromaticity & -5.24 & -4.00 \\
\hline Vertical Chromaticity & -7.40 & -14.82 \\
\hline Max. Horizontal Beta (m) & 5.62 & 2.63 \\
\hline Max. Vertical Beta (m) & 5.54 & 11.4 \\
\hline Max. Dispersion (m) & 0.62 & 0.54 \\
\hline Energy Loss per Turn* (MeV) & 0.617 & 0.617 \\
\hline RF Voltage (MV) & $\sim 1.2$ & $\sim 1.8$ \\
\hline RF Frequency $(\mathrm{MHz})$ & 1299 & 1299 \\
\hline Energy Spread (parts in 1000) & 1.52 & 1.52 \\
\hline Bunch Length* (mm) & 8.1 & 7.2 \\
\hline Horz. Damping Time* (ms) & 0.570 & 0.428 \\
\hline Vert. Damping Time* (ms) & 0.584 & 0.438 \\
\hline Energy Damping Time* (ms) & 0.295 & 0.222 \\
\hline Quantum Lifetime* (s) & $>1 \times 10^{10}$ & $1.5 \times 10^{9}$ \\
\hline Min. Physical Aperture* (sigma) & 11.0 & 13.4 \\
\hline
\end{tabular}

\section{DISCUSSION}

In order for the four cell lattice to work as simulated, the superconducting ring dipoles must have the following characteristics: 1) The magnet gap is more than four times smaller than the pole width. In our case, the dipole gap is $40 \mathrm{~mm}$ while the pole width is $180 \mathrm{~mm}$. 2) The magnetic field must be good to a few parts in ten thousand over $110 \mathrm{~mm}$ of the pole width. 3) The magnet must behave like an iron dominated magnet in that the magnetic length and the iron length must be nearly the same and the field must fall off rapidly at the ends of the magnet. In our case, the magnetic length is $405 \mathrm{~mm}$; the iron physical length is $380 \mathrm{~mm}$. (The coils at the end are about $32 \mathrm{~mm}$ longer than the iron.)
Three dimensional computer models of the dipole[2] show that the field can be made very uniform across the pole. The current in the coils can be adjusted so that the good field uniformity can be achieved from an induction of $0.432 \mathrm{~T}$ (corresponding to $100 \mathrm{MeV}$ injection) to $6.918 \mathrm{~T}$ (corresponding to a maximum ring energy of $1.6 \mathrm{GeV}$. The magnetic field falls from $6.5 \mathrm{~T}$ to $0.02 \mathrm{~T}$ in less than $20 \mathrm{~mm}$. The integrated field through the dipole is also very uniform. For a ring energy of $1.5 \mathrm{GeV}$ the dipole induction at the center is less than $6.5 \mathrm{~T}$.

In both the six cell ring and the four cell ring, the maximum pole induction for the quadrupoles was set to be about $0.6 \mathrm{~T}$. The maximum pole induction for the sextupoles is about $0.5 \mathrm{~T}$. As a result, the poles of the quadrupoles and the sextupoles can be shaped so that the magnets have a large good field region over the full range of machine energies.

The straight sections for both machines are short, but they are long enough for conventional $805 \mathrm{MHz} \mathrm{RF}$ cavities or $1300 \mathrm{MHz}$ superconducting cavities. In both the six cell and the four cell machine, the RF cavities would occupy one straight section. In both machines, the injection elements would occupy two cells. The remaining cells could contain either superconducting or conventional insertion devices. The beam energy is low enough to allow the beam to be dumped into the vacuum chamber.

\section{CONCLUSIONS}

It appears that a four cell $1.5 \mathrm{GeV}$ electron storage ring could be built to fit in the $9.142 \mathrm{~m}$ (30 feet) vault at UCLA. There is enough room in the four cell lattice for RF cavities, injection elements and perhaps an insertion device. The lattice has a long quantum life time, and the emittance is low enough to produce high intensity $\mathrm{X}$ ray beams for users. The $\mathrm{X}$ ray power and brightness is comparable to the six cell machine.

\section{ACKNOWLEDGMENTS}

The authors acknowledge discussions they have had with D. B. Cline and J. J. Kolonko of the UCLA Center for Advanced Accelerators. This work was performed with the support of the Regents of the University of California.

\section{REFERENCES}

[1] A. A. Garren and M. A. Green, "An Alternative Lattice Design for a Compact Light Source Ring," Proceedings of the 1997 Particle Accelerator Conference, p 520, IEEE Publications, New York (1997)

[2] M. A. Green and C. E. Taylor, "Three Dimensional Field Calculations for a Short Superconducting Dipole for the UCLA Ultra Compact Synchrotron, " to be published in the IEEE Transactions on Applied Superconductivity (1999) 